\title{
Software-Defined Wireless Transport Networks for Flexible Mobile Backhaul in 5G Systems
}

\author{
Dudu Bercovich, Ceragon, dudub@ceragon.com \\ Luis M. Contreras ${ }^{1}$,Telefónica, luismiguel.contrerasmurillo@telefonica.com \\ Yoram Haddad, Jerusalem College of Technology, haddad@jct.ac.il \\ Ariel Adam,Ceragon, ariela@ceragon.com \\ Carlos J. Bernardos, Universidad Carlos III de Madrid, cjbc@it.uc3m.es
}

\begin{abstract}
Traditionally microwave backhaul has been configured and operated in a static manner by means of vendor specific management systems. This mode of operation will be difficult to adapt to the new challenges originated by $5 \mathrm{G}$ networks. New mechanisms for adaptation and flexibility are required also in this network segment. The usage of a signaled control plane solution (based on OpenFlow) will facilitate the operation and will provide means for automation of actions on the wireless transport network segment. In addition to that, a standard control plane helps to reach the multi-vendor approach reducing complexity and variety of current per-vendor operation. This paper presents the motivation for the introduction of programmability concepts in wireless transport networks and illustrate the applicability of such control plane with two relevant use cases for dynamically controlling wireless transport nodes in $5 \mathrm{G}$ networks. Extensions to OpenFlow protocol are also presented for building Software Defined Wireless Transport Networks (SDWTNs).
\end{abstract}

\section{Keywords}

Wireless Transport Networks; Microwave; Software Defined Networking; Mobile Backhaul.

\section{Introduction}

After the tremendously increase of the mobile traffic due to smartphones the industry is now at the beginning of a new "storm" with the growing market of wearable devices such as connected watches, glasses and health wireless sensors. Existing cellular infrastructure was not originally designed to cope with such amount of traffic. Therefore lots of efforts have been invested to improve the capacity of the Radio Access Networks (RAN) lastly with the deployment of LTE-Advanced networks. However it seems that it will not be enough and further improvement will be mandatory to "survive" this new era. The advent of the new $5^{\text {th }}$ generation of mobile systems will impose new and stringent requirements to the existing transport networks. For instance small cells will be deployed almost

\footnotetext{
${ }^{1}$ Corresponding author.
} 
everywhere as one of the solution proposed. This kind of advanced architecture will put now the burden on the Mobile Backhaul (MBH) which was somewhat "forgotten" up to now.

The MBH networks are characterized by a large number of small/medium sites connected to centralized aggregation points in charge of concentrating traffic towards the core network. Several technologies can be used for backhaul, mainly microwave and optical transmission. Those different substrate technologies have distinct particularities in terms of performance, reliability and delay. All of them typically coexist in a real mobile network.

On top of that there is generally an Ethernet-based network providing packet switching transport capabilities. All this multi-layer and multi-technology approach presents some challenges at the time of providing a common and unified service across the backhaul segment. During service provision any action should be coordinated in time and manner for ensuring service consistency.

This MBH transport segment has to face a number of challenges derived from $5 \mathrm{G}$. Among those challenges are [1] the necessity of supporting high traffic volumes, the provision of high capacity on-demand for certain locations, and the capability for fast re-configurability. In addition to that, efficient management of the consumed energy and advanced $\mathrm{MBH}$ planning tools are also seen as relevant challenges.

As mentioned, the MBH in general and more specifically the microwave backhaul is a field that has not received too much attention from the research community. One of the possible reasons is that up to now most of the configuration is static. This was possible thanks to large spectrum bands still available, at that time, in the higher frequencies. However this perception is changing because of the appearance of new needs to be supported by the MBH networks.

The Software Defined Networking (SDN) paradigm [2] promotes the separation of the control plane capabilities from the data plane ones in contrast with the existing integration present in the network elements of the backhaul network, with a tightly coupling of both capabilities. With SDN the control plane is proposed to be logically centralized in a control entity with global responsibility for the control of the network elements in its domain. This global vision can facilitate the transversal task of orchestrating diverse network resources for composing and maintaining an end-to-end connectivity service.

$\mathrm{SDN}$ is positioned as one of the key technology trends that will facilitate the required architectural agility needed in the next 5G networks, especially in terms of enabling programmability and facilitating the separation of control and user plane functions [3]. Especially for mobile and wireless networks [4] several relevant challenges emerge for future architecture supporting 5G, with SDN standing as one of the key technologies capable of facing those challenges, bringing new technical and business opportunities for those future systems.

While some initiatives for control plane centralization are available nowadays for some parts of the network like the optical layer (e.g., based on GMPLS control mechanisms), the wireless transport technologies lack from such approach, relaying basically in the interaction with proprietary Network Management Systems (NMSs) for both control and management functions. 
This paper focuses on Wireless Transport Networks (WTNs) at the backhaul level and explores the promising control capabilities behind the emergence and application of the SDN concept to the wireless transport area for covering such gap. The paper structure is as follows. Section 2 presents the general motivation for the introduction of SDN control in the wireless transport segment. Section 3 presents two experiments that have been performed to check the suitability of this kind of technology. A brief review of other initiatives in the field of SDN for wireless and mobile networks is provided in section 4. Finally, in section 5 future directions and conclusions are reported.

\section{Motivation for Software Defined Wireless Transport Networks}

Typically, the technical implementation of transport equipment is very different among vendors. Since the functionalities are highly dependent of physical parameters each specific engineering solution does not allow an easy nor practical interoperability. This is particularly true in the case of WTNs, where the different approaches to solve the radiorelated issues makes each solution fine-tuned to work exclusively with a counterpart of the same vendor.

However, those limitations are not only present at physical level in the data plane. In general each manufacturer proposes different ways of managing and configuring their equipment. This fact complicates the operation of the WTNs, requiring specific procedures and skills to handle the network in a per-vendor fashion.

The specialization in the control of these networks produces a lack of agility that prevent for deploying advanced services over these infrastructures. There is no way of defining common actions or possibilities for dynamic provision of the WTN substrate for a whole network in a simple way.

SDN brings the opportunity to simplify the operation on one hand and allow the dynamic provision of advanced services on the other. The key concept to reach those goals is the idea of network programmability, which permits the instantiation of transport network capabilities on-demand and by means of common interfaces, usually in the form of Application Programming Interfaces (APIs). This is especially relevant in the WTN because of the lack of any other existing alternative to do that. Then from the application of SDN concepts to the wireless transport arena emerges the idea of Software Defined Wireless Transport Networks (SDWTNs).

\subsection{Problem statement}

As described before, mobile backhaul networks are composed of a variety of transport technologies, ranging from optical to wireless, apart from the packet-switched based aggregation riding on top of them.

In addition to that, from a service perspective, the $\mathrm{MBH}$ requires to support multiple services and applications with very different characteristics in terms of quality requirements, route forwarding and bandwidth capacity. This will be even more notorious with the appearance of $5 \mathrm{G}$ networks. 
The static provision of transport capabilities is no longer an efficient way of handling the network; neither the approach of segmenting the network per-technology and per-vendor to configure each of those segments in an isolated manner. A uniform control and management capability across all those segments is required to simplify the operation of new networks and to enable more efficient resource management such as the reutilization of unused resource from one operator to another, which is not possible with a local approach.

The control and management actions in SDN come from a logically centralized controller, keeping the logic to instruct the underlay devices in order to take actions in the data plane. Assuming a backhaul network fully composed of SDN-controllable nodes, one approach for the control architecture could be to consider the existence of a unique controller for all the nodes in the backhaul. This would require from that centralized entity to have control capabilities for handling a variety of technologies and family of nodes. Additionally, the existence of a large number of devices (due to the capillarity of the backhaul segment) complicates the operation and compromises the scalability of the controller. Furthermore, gathering of the network state toward a central point will likely lead to bottleneck in the vicinity of this controller. In consequence, the deployment of a single SDN controller in the backhaul segment to manage all the required connections does not scale.

Another approach is to consider the stacking of several controllers, one per layer, working together in a cooperative fashion. For instance, impacts on the effective modulation due to climatic conditions in the radio link of a microwave backhaul can be directly translated into traffic flow shaping actions (or even flow discard) deep in the network. This means that a very close coordination among controllers should be in place in order to collaborate in the service provision. In parallel, we can also consider a horizontal splitting of the controller authority where each controller is in charge of a limited area. For instance, in [5] different hierarchies and topology for set of controllers deployment is proposed. The ONOS (Open Network Operating System) project [6] is a good example of efforts to tackle the challenge of providing a centralized coordination meanwhile avoiding performance degradation at the control plane. ONOS maintains a global view of the network while SBI is physically distributed among multiple servers. Consistency of the database is kept through the use of open source module such Blueprints whereas fault tolerance is managed through redundant SBI links (i.e. switches connected to multiple ONOS instances) in a cluster like architecture with only one master at a time.

From the perspective of a controller devoted to the WTN part, such coordination will be delivered through a North Bound Interface (NBI) connecting to a controller on top of it, probably with orchestration capabilities across domains and technologies in the $\mathrm{MBH}$. Additionally, the WTN controller will interact with the microwave network elements via a common South Band Interface (SBI) for injecting rules and providing control and management instructions.

\subsection{Open Network Foundation initiative for wireless transport networks}

The Wireless and Mobile Working Group (WMWG) in the Open Network Foundation (ONF) is working on the specification of OpenFlow protocol extensions for SDN control of WTNs. 
OpenFlow [7] specifies a common standard interface between the controller and forwarding network element in SDN and is therefore one possible (maybe the most famous up to now) instantiation of the SBI. Because of its vendor-neutral conception, the usage of OpenFlow facilitates the integration of heterogeneous technical implementations of WTN nodes from the control point of view simplifying the operation of multi-vendor infrastructures.

Several use cases are being considered on the scope of the WMWG, such as dynamic radio resource management, unified management of SDWTN nodes, or efficient management of power consumption.

The WMWG is currently defining an SDN architecture suited to the WTN according to a functional model of microwave systems, considering simple, hybrid and multi-radio cases. In the case of simple radio the same kind of traffic is transported over a single microwave radio link. In the hybrid case different types of traffic are transported using the same link. And finally, the multi-radio case address the situation in which a certain traffic type is carried on several radio links.

All of this is formalized through the definition of a microwave-related information model to support the necessary abstraction to homogenize control procedures. As result of such architecture the next steps consists on deriving OpenFlow requirements that finally can be materialized as extensions to the protocol.

The referred extensions will be needed for handling a number of parameters present in wireless transport systems. Apart for being able to configure such parameters, it is also required to collect the status and to provide notifications on changes that can simplify the management of such systems.

The extensions being into consideration refer to both physical and logical parameters. By physical parameters, the idea is to access and control actual physical metrics of the microwave equipment such as transmission power, link degradation, latency, etc. On the other hand, the concept of logical parameters refers to logical parameters describing a point-to-point radio physical link, such as capacity, availability, etc. While the physical ones are directly read from the microwave equipment components, the logical ones requires some processing by the equipment itself (e.g., data aggregation).

\subsection{Related work}

The changes expected at the RAN with the advent of $5 \mathrm{G}$ networks give rise to challenging issues at the MBH level that stress the need for a flexible and more efficient MBH. Such flexibility can be potentially achieved with the emerging new concept of SDN.

For instance, in the last years there has been an increasing interest in the context of ultradense networks (UDN) to be deployed for satisfying the ever increasing bandwidth demand on the mobile network. Such UDN will require multitude of small cells which classical point to point backhaul is no longer a viable and scalable solution. Some works have proposed some alternatives to this new challenge. In [8], the use of the millimeter wave (VBand and E-Band) is proposed to provide backhaul to the increasing number of picocells. This band enjoys generous spectrum availability and therefore does not require a priori complex techniques such as cross channel polarization, channel aggregation, etc. Centralized and cross-layer control mechanisms appear as necessary for fully exploiting the capabilities offered by the forwarding plane of millimeter waves [9]. In [10], a dynamic 
resource allocation of an OFDMA based backhaul to small cell is considered. An algorithm for downlink scheduling and power allocation is proposed. It is worth to note that their proposed scheme assumes the existence of a server that has a real-time global knowledge of the channel state information and allowed interference level. This latter entity reminds interestingly the concept of software driven control.

Another solution proposed to improve RAN performance is the Cloud-RAN (C-RAN) concept [11]. In this case a new kind of backhaul appears (referred to as fronthaul). Some recent works [12] analyze the way for this distributed set of remote units (known as Remote Radio Head) to be connected to the baseband unit where in some cases a joint optimization of RAN and backhaul could be considered [13].

The potential benefit of introducing SDN into the wireless and mobile world has already been identified in several works in the last years $[14,15,16]$. However few works have explicitly identified the opportunities of SDN to improve mobile backhaul performance. One of the few first works dealing with this is [17]. The authors propose to avoid static resource allocation through a Backhaul Resource Manager (BRM). This BRM is actually a controller like entity with global knowledge of the link state. This knowledge enables also in a second step to implement a capacity-aware path computation where surprisingly sometimes longer path should be selected over shorter path to avoid congested link. However no practical insight on the implementation of these ideas was provided.

The work in [18] presents and OpenFlow-based packet switched backhaul where simple Ethernet switches are used, moving the intelligence associated to the mobile network to external applications interacting with a centralized SDN controller.

In [19] SDN is integrated with a Wireless Mesh Networks (WMNs) in backhaul where the entire WMN is abstracted appearing to the controller as if it was a single switch, then simplifying its control.

The EU CROWD FP7 project proposed in [20], a comprehensive architecture based on SDN for cellular and WLAN technologies. The control plane is hierarchically organized into two tiers where "districts" have a local knowledge for short time scale decision and "region" have global information for more coarse grain long time scales In addition two North Bound APIs type are proposed namely: Technology Specific where optimization is performed on details specific to a given technology (e.g., InterFrame Space for WiFi AP) and Technology Agnostic which enable generic optimization on broad range of technology based on set of abstracted primitives. Finally reconfigurable backhauling is considered with special emphasis on the opportunity given by SDN for traffic-proportional backhaul configuration through the controller.

\section{Use cases for SDWTN}

As previously mentioned, 5G systems will impose a number of challenges to the transport networks. Apart from on-demand bandwidth capabilities and efficient power consumption, programmability for fast re-configurability is one of the major needs for transport networks.

In this section we will describe two applications to illustrate the resource optimization of WTN using SDN. The first application is selective traffic shaping and reroute, and the second one is dynamic spectrum sharing. 


\subsection{Selective Traffic Shape and Re-route}

Wireless links have a number of special attributes and among them is the fact that the links "breath". This means that the links capacity can change depending on the weather and environment conditions such as rain.

In the traditional WTN, the capacity degradation is handled by statically applying QoS techniques which basically drop packets locally based on their class of service.

SDN provides the opportunity to handle this situation in a more optimal and dynamic way. For instance, if we consider a centralized controller maintaining a global view of the whole network, in case there is a capacity degradation event it is possible to leverage on this to take actions deeper in the network.

The controller can identify the flows that are supposed to go through the degraded link, and apply shaping on them by instructing network elements (the ones of WTN or others in the core). In other words the system can command ahead of time and upfront, the source of this flow to stop, for a moment, transmitting it. This way it is possible not only lowering the burden on this degraded link but also avoiding the data of this flow from being discarded too late after having achieved sometimes "a long trip" toward the bottleneck and thus wasting the resources of the intermediate nodes as well. It can even reroute some of the flows to new routes that have available capacity.

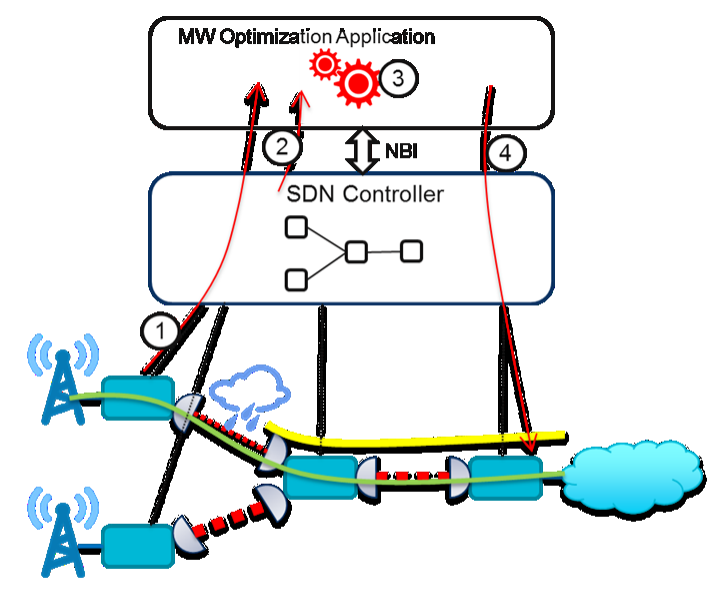

(a)

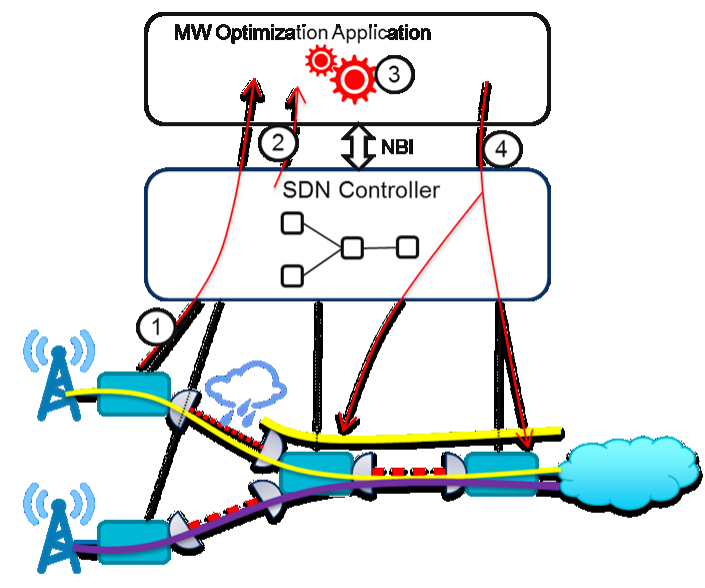

(b)

Figure 1. Selective traffic shaping (a) and re-routing (b).

According to Figure 1, the sequence of actions to perform the resource optimization is the following:

1. Because of a degradation in the transmission conditions of the radio part (e.g., due to rain), an event for change in the capacity link is notified to the optimization application, via the SDN controller.

2. The application generates an updated view of the topology and the flows riding on top of it. 
3. With the previous information, the application calculates the optimal point for applying shaping for the selected flows, in such a way that the resulting global resource usage becomes optimal.

4. At the end of this process, the application instructs the SDN controller for populating the necessary rules in the intended network elements for committing the necessary changes.

In the above Figure 1(a) it can be seen that shaping is applied to the "yellow" flow to match the available end to end capacity, resulting in the less bandwidth consuming "green" flow. The same approach is applicable for different actions, like traffic diversion on alternative paths if available, as shown in Figure 1(b), increasing the amount of traffic of the "purple" flow as in the example.

In this scenario the granularity in the flow handling can be further enhanced by applying Deep Packet Inspection (DPI). DPI permits to look deep into the L2/3 flows, even identifying specific applications. This allows applying actions per service application. The controller can decide to shape specific applications, and reroute others. For example, time sensitive traffic and/or premium customers could be granted with priority, forwarding its corresponding traffic over the residual bandwidth of the degraded (but preferred) link whereas background traffic can be rerouted over an alternative path that could suffer in some cases higher delays.

\subsection{Dynamic Spectrum Allocation}

In regular microwave point-to-point planning two links that are aggregated at the same point need to use different channels if the angle of separation between the two aggregating antenna at the aggregation node is not large enough (more than 90 degrees). In Figure 2, it can be shown two different base stations (namely $B S 1$ and $B S 2$ ) connected via MW links to the aggregation node $A N$. The backhaul link 1 for $B S 1$ is directed to antenna 1 (ant.1) and link 2 for BS2 to antenna 2 (ant.2). In this case there are only 15 degrees of separation between the antennas, which requires two completely separated channels namely $f 1$ and $f 1$ ' for DL and UL of link 1 respectively and $f 2$ and $f 2$ ' for DL and UL of link 2 resp.

The advantage of this planning method is that even if there strong interference conditions where one of the links suffers from fade while the second one does not experience degradation, the complete isolation of the two channels ensure a stable CIR. 


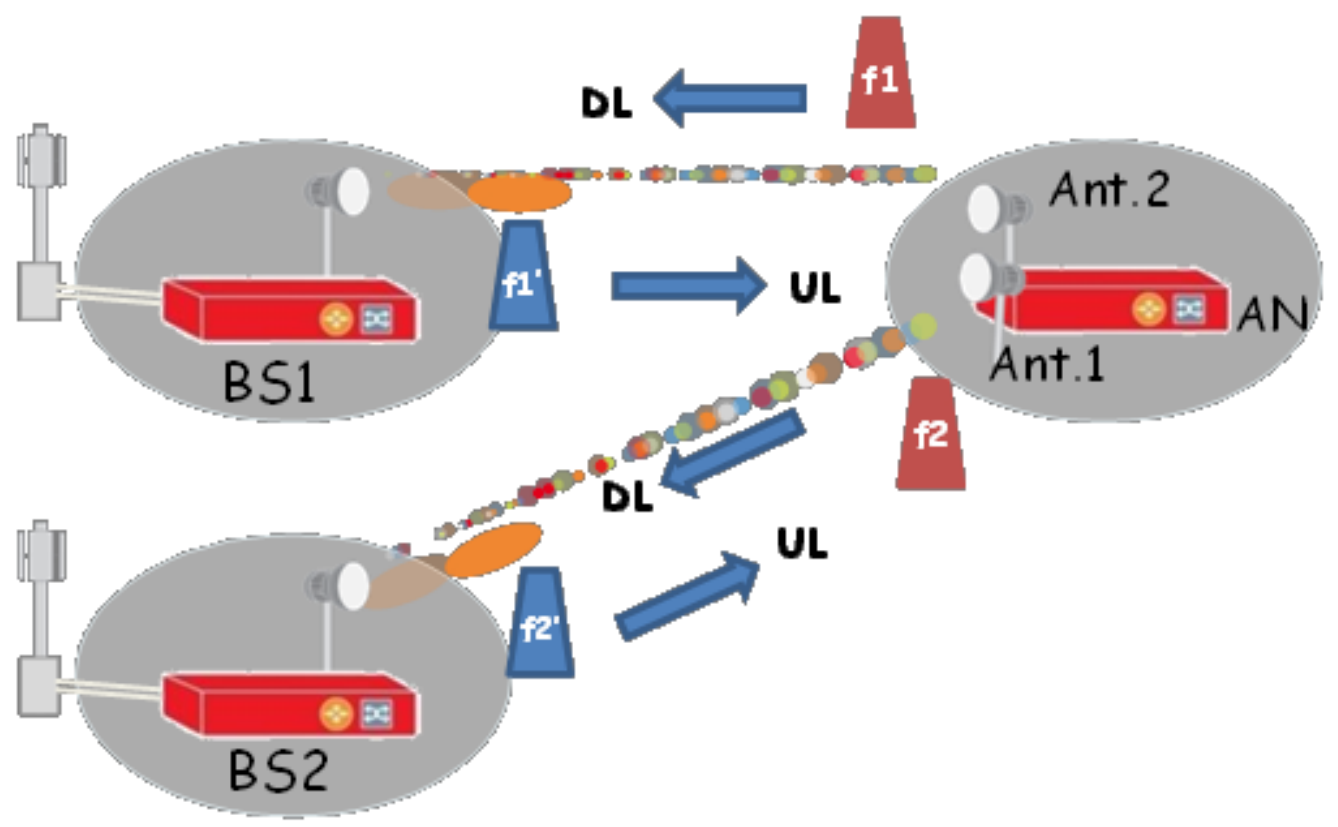

Figure 2. Low spectral efficiency case.

However the aforementioned planning leads to low spectral efficiency. Most of the time even smaller angles in the antenna separation (such as 30 degrees) are enough to allow both links to share the same frequency. In Figure 3 we show a similar scenario to Figure 2 but with antenna separation at $A N$ of 30 degrees which enables both link to use the two channels. Let's assume, for the purpose of this example, that a required CIR of $40 \mathrm{~dB}$ is satisfied in both links.

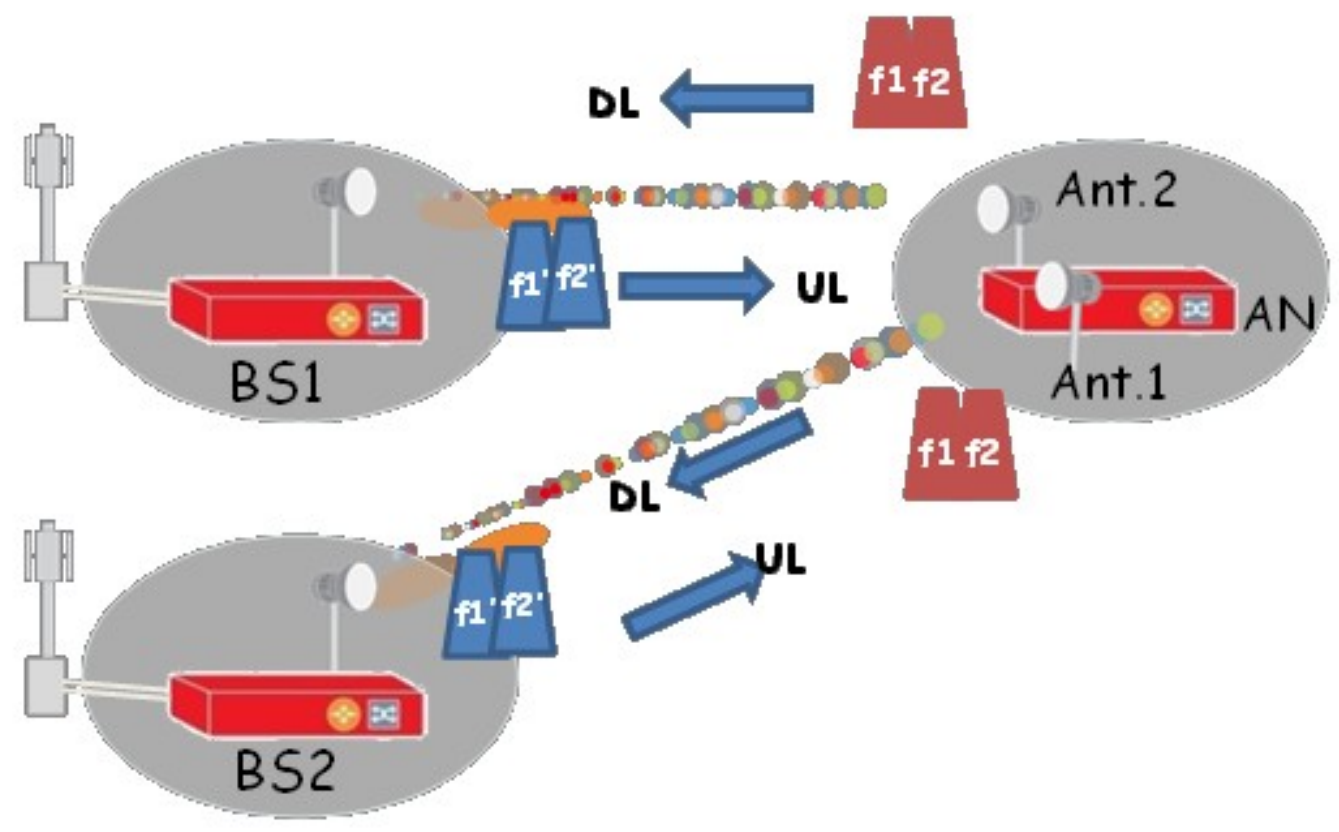

Figure 3. Full frequency reuse due to enough antenna separation 
A problem can occur when there is an asymmetrical fading situation. In this case, link 1 undergoes a fading due to weather condition whereas in the same time link 2 has excellent condition. The problem arising is that the interference generated from $B S 2$ on link 1 will now be relatively stronger due to a decreasing received signal from $B S 1$ at Ant.1 which will lead to a link 1 UL CIR decreased, let's say now equal to $20 \mathrm{~dB}$.

One of the possible (not optimal solution) would be in this case to have the channel arrangement fall back to the original planned allocation. The best solution however will be based on the programmability offered by SDN. A spectrum allocation system that sits at the central controller will dynamically allocate channels to the links based on interference status with a priority based paradigm. Figure 4 schematically represents this situation. As soon as the degradation due to weather condition is detected by Ant.1 an event is announced at the SDN controller. Then the controller (after running some spectrum allocation algorithms likely to be implemented by an application on top of the controller) will configure the BS2 as following. The power transmitted of BS2 on channel $f 1$ ' is reduced by a factor of $20 \mathrm{~dB}$. This will allow the CIR of channel $f l^{\prime}$ of link 1 to be maintained at $40 \mathrm{~dB}$.

Also on demand schemas can be supported, providing the system with rich flexible capabilities to serve dynamically traffic needs.

Each link gets the right to use a channel marked as high priority. In addition the Spectrum allocation system can allocate a second channel with a lower priority.

The spectrum allocation system is aware of the reception signal quality for all the links in a region, and it can detect interferences if they occur. When the spectrum allocation system detects signal interference in a link on its priority channel, the system automatically requests the other links that previously got permission to use this channel as lower priority link to cease using it. This way the link which has the priority over this channel can use it without any degradation.

Simulation analysis results of these techniques for urban scenarios [21] have shown that it is feasible to use half of the spectrum with respect to regular planning methods, reducing from 24 channels of $7 \mathrm{MHz}$ to just 12 with spectrum sharing. SDN-based control can provide such a relevant efficiency in an automated and dynamic way. 


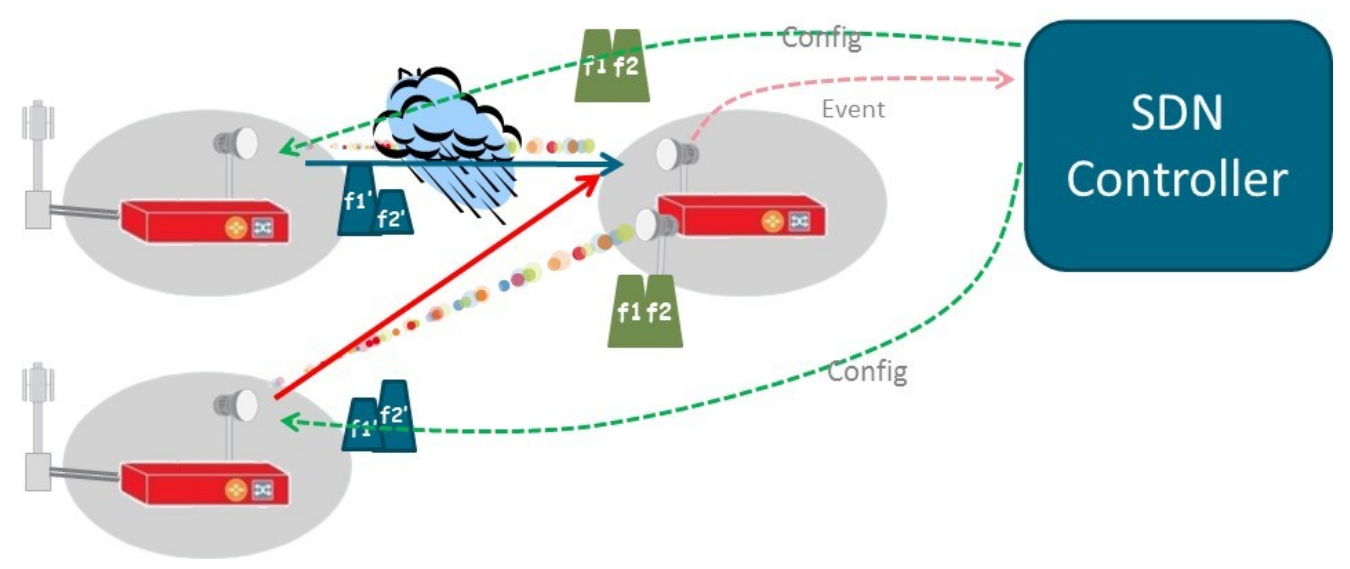

Figure 4. Dynamic spectrum allocation

The effectiveness of the solution proposed above can be validated by the following measurement. We still consider the scenario presented in Figure 2. We consider in our experiment two possible antennas namely $1 \mathrm{ft}$ and $2 \mathrm{ft}$ antenna transmitting and receiving at $18 \mathrm{GHz}$. In figure 5 and 6 we display the radiation pattern of $1 \mathrm{ft}$ and $2 \mathrm{ft}$ antenna respectively. In these figures we show the measured radiation decrease relatively to the maximum signal received when the receiver is located at a given azimuth degrees (varying on the $\mathrm{X}$ axis) from the main lobe of the transmitter. Obviously the reception is maximal when the angle between the direction of the lobe of the transmitter and the receiver is 0 .

In each of the figures we present 4 possible combination of polarization (vertical polarization denoted $\mathrm{V}$ and horizontal $\mathrm{H}$ ). The blue upper curve depicts the radiation pattern when a $\mathrm{V}-\mathrm{V}$ transmission-reception polarization is considered. The red upper curve is for the $\mathrm{H}-\mathrm{H}$, blue bottom curve for the $\mathrm{V}-\mathrm{H}$ and the red bottom curve for $\mathrm{H}-\mathrm{V}$.

It is clear the received signal strength is maximal when co-polar $(\mathrm{V}-\mathrm{V}$ or $\mathrm{H}-\mathrm{H})$ is considered whereas cross-polar scenario ( $\mathrm{V}-\mathrm{H}$ or $\mathrm{H}-\mathrm{V})$ yields to an automatic $30 \mathrm{~dB}$ degree even if the received is focused on the main lobe of the transmitter. 


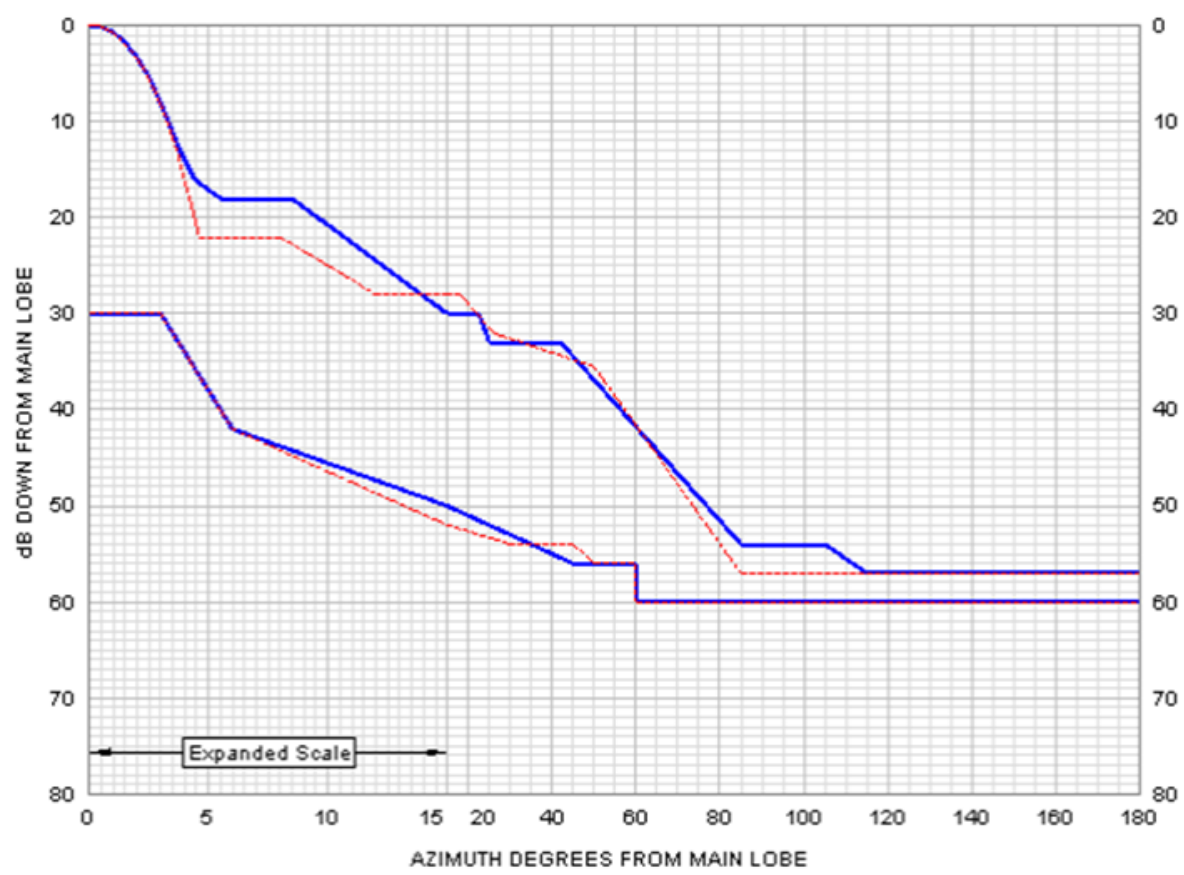

Figure 5: $1 \mathrm{ft}$ antenna radiation pattern at $18 \mathrm{Ghz}$.

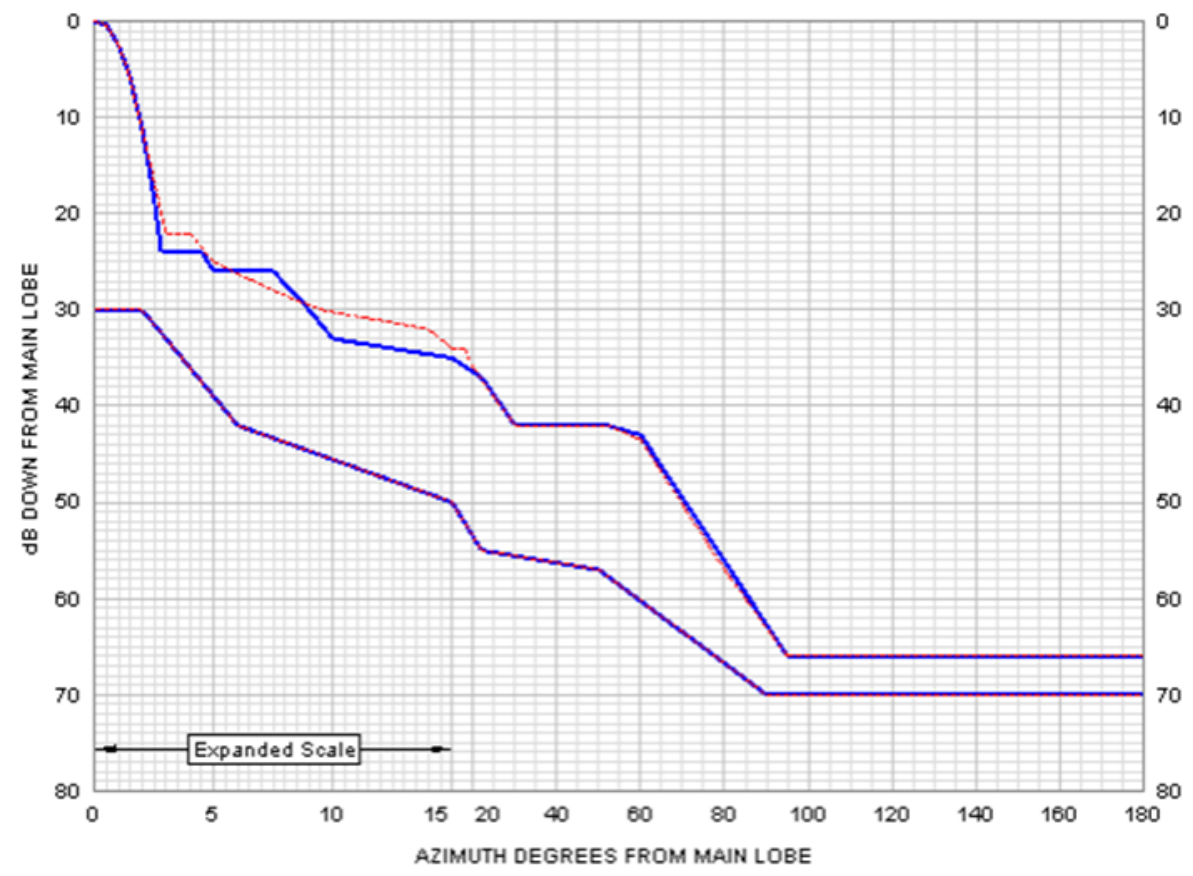

Figure 6: $2 \mathrm{ft}$ antenna radiation pattern at $18 \mathrm{Ghz}$. 
Now, let's assume as previously that the required CIR is $40 \mathrm{~dB}$ and also assume sporadic fading of $20 \mathrm{~dB}$. If we consider regular planning without using SDN we see on the $1 \mathrm{ft}$ antenna graph (Fig. 5) that a separation of at least 120 degree in the co-polar case and 60 degree in the cross polar one will be required in order to ensure a CIR of $60 \mathrm{~dB}$. In the case of a $2 \mathrm{ft}$ antenna if will be 85 and 60 for the co-polar and cross polar case respectively. So in case of a static (non SDN) resource allocation, the same frequency can be reused with a minimum of 60 degrees separation (assuming cross polar) which constitute a serious limitation if the BS to backhaul are too close.

If we consider now the scenario depicted in Fig.4 where a SDN controller hold up to date information regarding the links, can detect a fading event and in consequence trigger some changes on the way the transmission is performed between BSs and AN we can then reuse the same frequency with a CIR of only $40 \mathrm{~dB}$ which correspond to a 55 degree of separation for a $1 \mathrm{ft}$ antenna and only 25 degrees for a $2 \mathrm{ft}$ antenna both in the co-polar case.

If a fading event occur we will then tackle whether by reducing the BW as proposed earlier in this section or by allowing each link using only one of the polarization (different from the other link) which will be bring us to the cross-polar case which almost provide what is required due to the fading decrease.

\section{Future directions and conclusions}

The wireless backhaul segment has been traditionally operated and configured in a static manner. The promise of programmability can open interesting opportunities in this part of the network, not yet explored in deep.

Apart from the experiments described before there are a number of some other interesting use cases that can be enabled by SDN. Aspects like energy efficient backhaul operation or multi-technology (e.g., packet-switched and wireless) coordination can be further exploited in a dynamic manner in a software driven control environment.

The former can be performed by adaptively configuring in time WTN equipment to consume just the required power according to the existing climatic conditions (e.g. reducing transmit power in good situation) or workload (e.g. using only the necessary radio resources in $\mathrm{N}+\mathrm{N}$ configurations) along the time.

The latter can leverage on the separation between logical or service connectivity from physical or transport connectivity. At logical level the constraints are expressed in terms of capacity, utilization, latency, availability, packet delay variation (PDV), packet loss (PL) and general cost functions (user preferences). In contrast, at physical level the constraints are related to link power consumption, spectrum frequency, modulation, receive signal level (RSL), etc. Additionally, the logical connectivity usually exceeds the scope of the mobile backhaul, extending the connection from access to the network core, and results agnostic about the conditions experienced by the underlying network. An effective coordination between all the network segments traversed, taking profit of reconfigurability and adaptability on the underlying transport capacity, can permit a joint optimization of the resources.

This duality is conditioned as well by the network topology. When planning a network rollout, the final footprint of the network capillarity depends on several factors, like 
infrastructure availability or radio frequencies in use. Considering one aggregation node and a given number of cellular sites, an optimal way to partition the network in rings can be defined, attaching each ring to the aggregation node. Once the network is deployed in this way it can suffer from some events or even it can evolve in time (e.g. because new cellular sites are commissioned) requiring automatic reconfiguration.

Increasing the number of rings can reduce the maximum path length and in consequence the unavailability. On the other hand, it requires more antennas. For any case, given the number of rings, it is preferable that the variance of the ring size distribution be as small as possible and that the largest ring be as small as possible. By introducing a cost function, we can prove that when the cost is more sensitive to failure probability, the optimal solution is to increase the number of rings.

Optimal topology, such as optimal ring size as mentioned, is possible only if there is access to real time link status. For instance if a link is degraded with increase failure probability then a re-arrangement of the rings will be necessary to maintain an overall acceptable availability (a.k.a. five 9's). Thanks to SDN, which enables the nodes to provide the control plane with up-to-date state channel link information, it is possible develop a dedicated application on top of the controller to manage dynamically the optimal topology. It should be mentioned that this assumes some kind of flexible antenna mechanisms (such as motorized rotating antenna by remote control) already existing for TV antenna and expandable to microwave backhaul.

The referred multi-technology coordination based on SDN will typically require the definition of the interconnection interfaces between per-technology controller, in terms of APIs, that can make possible a simple interaction among them. Capabilities such as topology exposition and network abstraction are needed to facilitate the orchestration of resources end-to-end. Additionally a simple but complete information model has to be defined for handling with multi-vendor scenarios from a common control system in the network.

SDN can also act as facilitator to use wireless transport equipment for directly connecting the radio base stations. Since all the control logic resides in the controller, wireless backhaul nodes can be potentially configured with a rich set of functionalities in the data plane level in principle without limitations from the control plane point of view.

In conclusion, SDN brings new dynamic control capabilities to WTNs that will permit the flexible operation of this network segment traditionally provisioned in a static pipe-like manner. The increase in the number of access nodes (e.g. because of small cell deployments) will motivate the need for reconfiguration and adaptation. All these changes can be facilitated with a homogeneous and dynamic programmable control as offered by SDN.

\section{References}

[1] Fiorani M, Monti P, Skubic B, Martensson J, Valcarenghi L, Castoldi P, Wosinska L (2014) Challenges for 5G Transport Networks. In: Proceedings of the IEEE International Conference on Advanced Networks and Telecommunication Systems. New Delhi, India, pp. 1-6. 
[2] Kreutz D, Ramos FMV, Esteves Verissimo P, Esteve Rothenberg C, Azodolmolky S, Uhlig S (2015) Software-defined networking: A comprehensive survey. Proceedings of the IEEE 103 (1): 14-76.

[3] NGMN Alliance (2015) 5G White Paper. https://www.ngmn.org/uploads/media/NGMN 5G White Paper V1 0.pdf

[4] Yang M, Li Y, Jin D, Zeng L, Wu X, Vasilakos AV (2015) Software-Defined and Virtualized Future Mobile and Wireless Networks: A Survey. Mobile Netw Appl 20:4-18.

[5] Schmid S, Suomela J (2013) Exploiting locality in distributed SDN control. In: Proceedings of the second ACM SIGCOMM workshop on Hot topics in software defined networking (HotSDN '13). Hong Kong, pp. 121-126.

[6] Berde P, et al. (2014) ONOS: towards an open, distributed SDN OS. In: Proceedings of the third ACM Workshop on Hot topics in software defined networking (HotSDN '14). Chicago, pp. 1-6.

[7] McKeown N, Anderson T, Balakrishnan H, Parulkar G, Peterson L, Rexford J, Shenker S, Turner J (2008) OpenFlow: enabling innovation in campus networks. SIGCOMM Computer Communication Review 38(2): 69-74.

[8] Sooyoung H, Taejoon K, Love DJ, Krogmeier JV, Thomas TA, Ghosh A (2013) Millimeter Wave Beamforming for Wireless Backhaul and Access in Small Cell Networks. IEEE Transactions on Communications 61 (10) pp.4391-4403.

[9] Niu Y, Li Y, Jin D, Su L, Vasilakos AV (2015) A survey of millimeter wave communications (mmWave) for 5G: opportunities and challenges. Wireless Networks, 1-20.

[10] Marić I, Bostjancic B, Goldsmith A (2011) Resource allocation for constrained backhaul in picocell networks. In: Information Theory and Applications Workshop (ITA), pp.1-6.

[11] China Mobile Research Institute (2011) C-RAN - Road Towards Green Radio Access Networks. http://labs.chinamobile.com/cran/wpcontent/uploads/CRAN_white_paper_v2_5_EN.pdf.

[12] Bartelt J, Fettweis G, Wubben D, Boldi M, Melis B (2013) Heterogeneous Backhaul for Cloud-Based Mobile Networks. In: 2013 IEEE 78th Vehicular Technology Conference (VTC Fall), pp.1-5.

[13] Bernardos CJ, Domenico AD, Ortin J, Rost P, Wübben D (2013) Challenges of designing jointly the backhaul and radio access network in a cloud-based mobile network. In: Proc. Of the Future Network \& Mobile Summit, Lisbon.

[14] Bernardos CJ, de la Oliva A, Serrano P, Banchs A, Contreras LM, Hao J, Zúñiga JC (2014) An architecture for software defined wireless networking. IEEE Wireless Communications 21 (3):52-61.

[15] Chaudet C, Haddad Y (2013) Wireless Software Defined Networks: Challenges and opportunities. In: 2013 IEEE International Conference on Microwaves, Communications, Antennas and Electronics Systems (COMCAS) pp.1-5. 
[16] Trivisonno R, Guerzoni R, Vaishnavi I, Soldani D (2015) SDN-based 5G mobile networks: architecture, functions, procedures and backwards compatibility. Transactions on Emerging Telecommunications Technologies 26:82-92.

[17] Bojic D, Sasaki E, Cvijetic N, Ting W, Kuno J, Lessmann J, Schmid S, Ishii H, Nakamura S (2013) Advanced wireless and optical technologies for small-cell mobile backhaul with dynamic software-defined management. IEEE Communications Magazine 51(9):86-93.

[18] Costa-Requena J, Kantola R, Llorente J, Ferrer V, Manner J, Yi Ding A, Liu Y, Tarkoma S (2014) Software Defined 5G Mobile Backhaul. In: Proceedings of the 1st International Conference on 5G for Ubiquitous Connectivity.

[19] Seppänen K, Kilpi J, Suihko T (2014) Integrating WMN based mobile backhaul with SDN control. In: International Conference on Software-Defined and Virtualized Future Wireless Networks (SDWN).

[20] Ali-Ahmad H, Cicconetti C, de la Oliva A, Draxler M, Gupta R, Mancuso V, Roullet L, Sciancalepore V (2013) CROWD: An SDN Approach for DenseNets. 2013 Second European Workshop on Software Defined Networks (EWSDN), pp. 25-31.

[21] CEPT ECC Report 211 (2014) Technical assessment of the possible use of asymmetrical point-to-point links.

http://www.erodocdb.dk/Docs/doc98/official/pdf/ECCREP221.PDF 